Supporting Information

for

\title{
Hierarchically porous composite scaffold composed of SBA-15 microrods and reduced graphene oxide functionalized with cyclodextrin for water purification
}

Youngjin Choi,${ }^{\dagger}$ Arjyabaran Sinha,${ }^{\dagger}$ Jihye Im ${ }^{\dagger}$ Hooyeon Jung, ${ }^{\dagger}$ and Jaeyun Kim ${ }^{*}, \dagger, \uparrow$,

${ }^{\dagger}$ School of Chemical Engineering, ${ }^{\star}$ Department of Health Sciences and Technology, Samsung Advanced Institute for Health Sciences \& Technology (SAIHST), ${ }^{\S}$ Biomedical Institute for Convergence at SKKU (BICS), Sungkyunkwan University (SKKU), Suwon 16419, Republic of Korea

* To whom correspondence should be addressed: Jaeyun Kim, kimjaeyun@skku.edu

Telephone: +82-31-290-7252. Fax: +82-31-290-7272

Y.C. and A.S. contributed equally to this work 

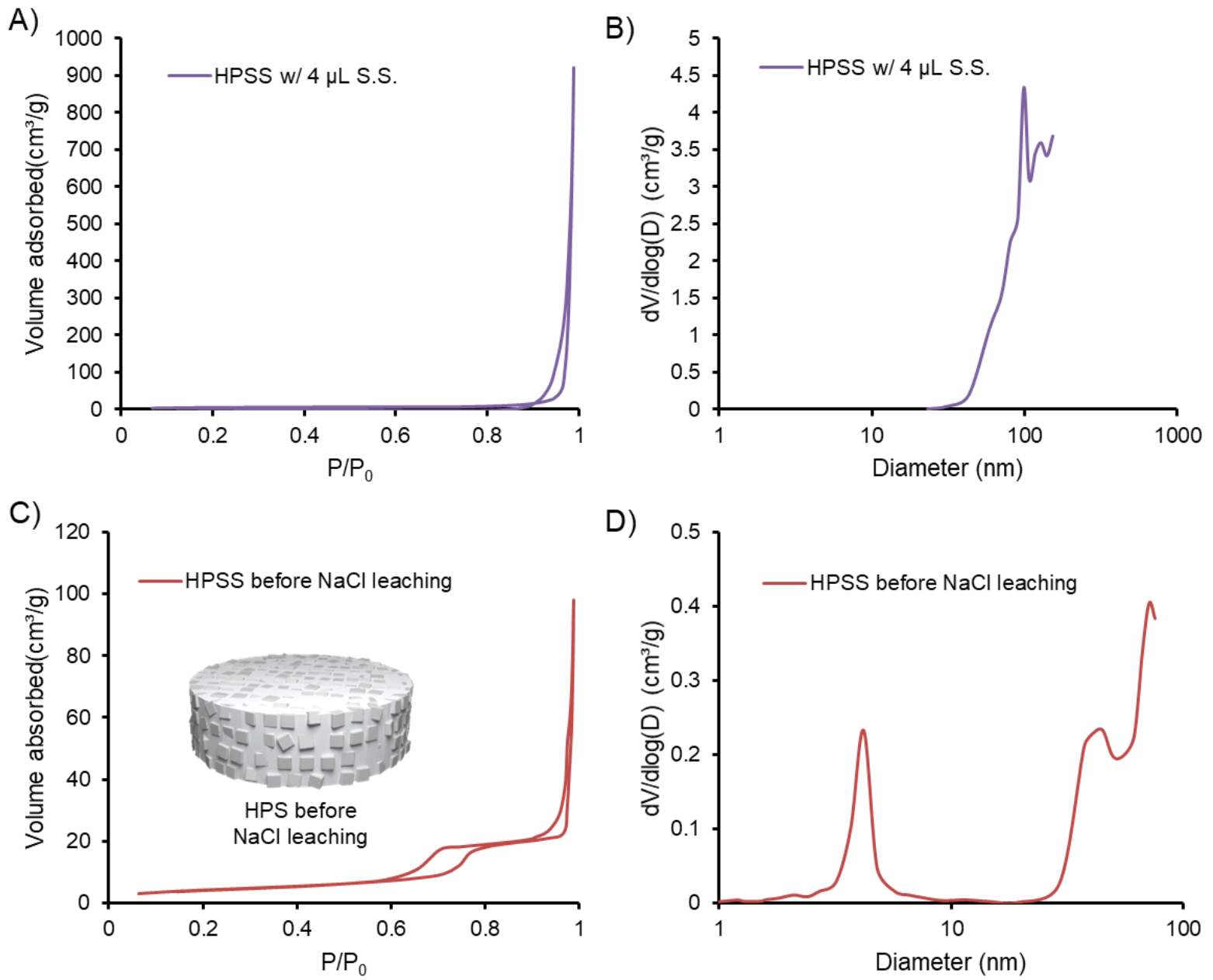

Figure S1. (A, C) Isotherms and (B, D) pore size distributions for nitrogen gas sorption of (A, B) HPSS prepared with excess amount of sodium silicate $(4 \mu \mathrm{L})$ and $(\mathrm{C}, \mathrm{D})$ the HPSS before $\mathrm{NaCl}$ leaching. 


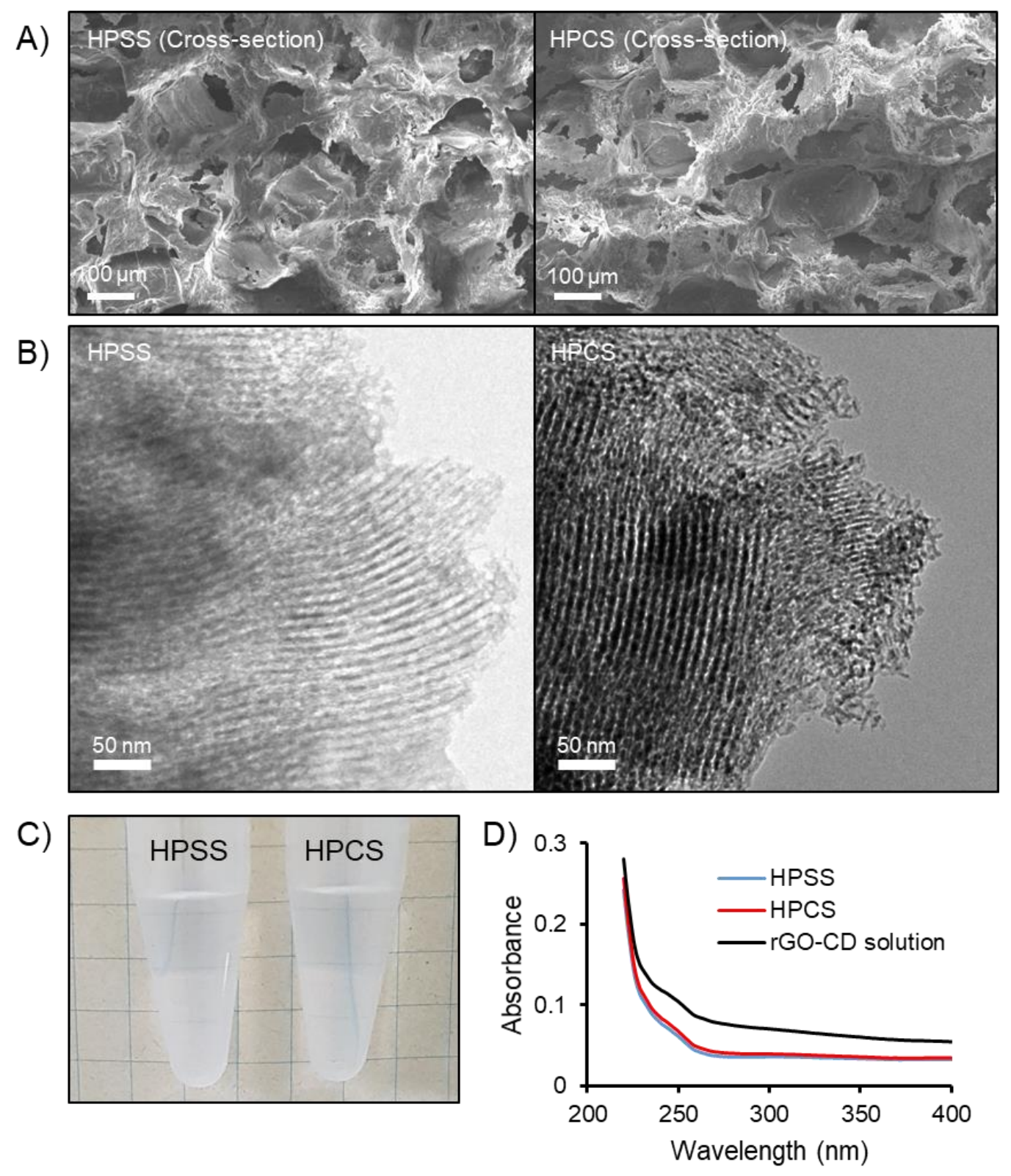

Figure S2. (A) Scanning electron microscopy (SEM) and (B) transmission electron microscopy (TEM) images of particles retrieved from HPSS and HPCS loaded with $800 \mu \mathrm{g}$ rGO-CD. rGOCD release during $\mathrm{NaCl}$ leaching step. (C) Photograph and (D) UV-Vis spectra of supernatant obtained during salt-leaching in the preparation of HPSS and HPCS, representing there was no significant release of rGO-CD during salt-leaching step. 

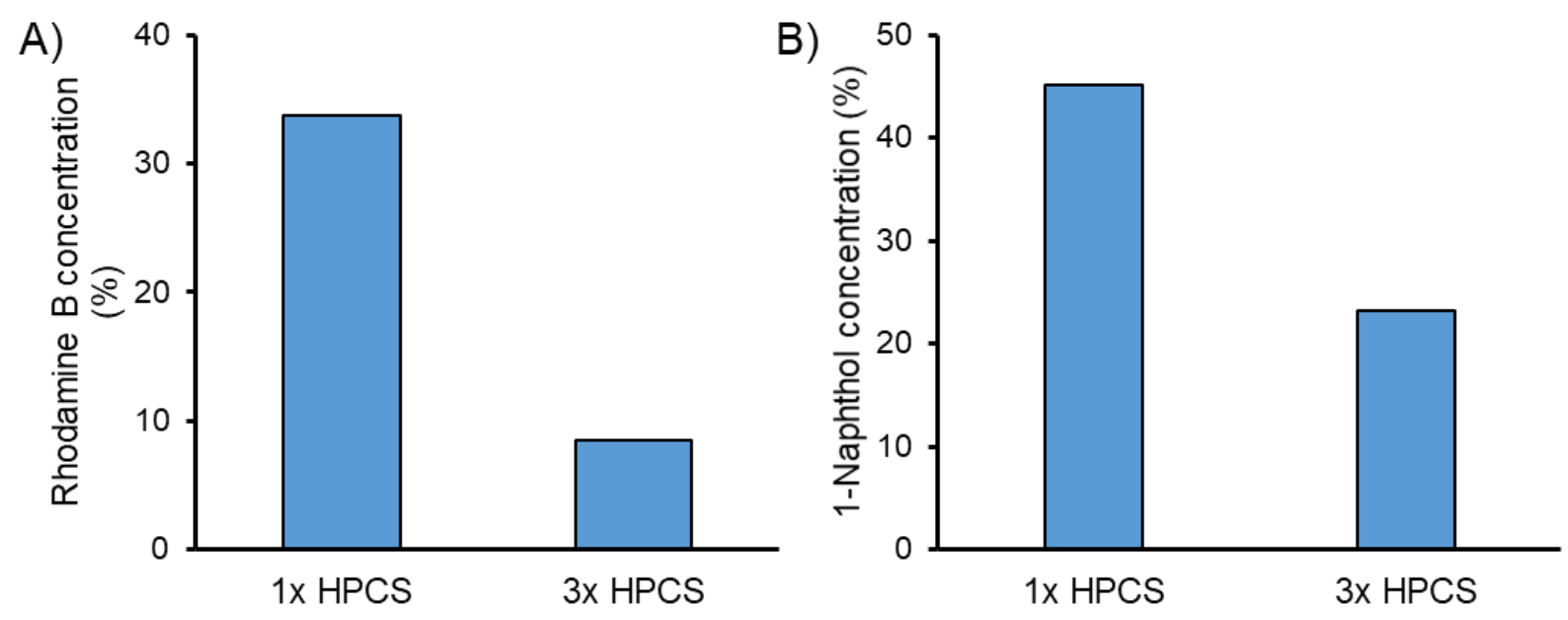

Figure S3. The concentration of (A) Rhodamine B and (B) 1-Naphthol in filtered solution based on batch-type separation with single HPCS (1x HPCS) and triple HPCS (3x HPCS) for $24 \mathrm{~h}$. 